\title{
Immediate and continuous processing of words in sentence comprehension
}

\author{
IN-MAO LIU \\ Chinese University of Hong Kong, Shatin, N. T., Hong Kong \\ and \\ JEI-TUN WU \\ National Taiwan University, Taipei, Taiwan, Republic of China
}

\begin{abstract}
In order to interpret eye-fixation data in reading, it is commonly held that an encountered word is processed as far as possible without its being reprocessed when a following word is encountered. Contrary to this assumption, this study found that, when a sentence-sentence verification task is used, a previous word receives further processing when a following word is encountered. This finding, however, by no means contradicts eye-fixation data obtained previously, because such data have relied on statistical averages and have not asked the question of how a particular word is processed in a particular position of a sentence.
\end{abstract}

In comprehending a simple sentence of the subjectverb-object type, there are at least two conceivable models. According to the immediate-processing model, a word is processed as far as possible immediately after its being encountered. No further processing is required when a following word is processed except that some representation of the previous word is integrated at the time of processing a following word. The immediateprocessing model is similar to the immediacy and eyemind assumptions (Just \& Carpenter, 1980) that form the current bases for interpreting eye-fixation data in reading. The immediacy assumption maintains that a reader tries to interpret each content word of a text as it is encountered and that the interpretations at all levels of processing are not deferred. The eye-mind assumption posits that there is little lag between what is being fixated and what is being processed.

According to the continuous-processing model, a word is processed only to some extent immediately after its being encountered and receives further processing at the time of encoding the following words. There are two advantages for this type of continuous processing. The first advantage is that, within the multiple-processing capacity of an individual (Navon \& Gopher, 1979), there results a saving in the reading time because partial processing of a previous word until the time of encoding a following word is delayed. The second advantage is that delaying partial processing of a previous word tends to facilitate integration or comprehension of a sentence because several words are processed simultaneously.

This study was supported by National Science Council Grant NSC-72-0301-H002, Republic of China. The authors are grateful to Michael H. Bond and King-leung Kong for their constructive reviews of earlier versions of this manuscript.
The present study was conducted to test whether the immediate-processing or continuous-processing model holds in reading. Let a simple sentence of the subjectverb-object type be denoted by S-V-O. In the present study, then, sentences of $S-V^{\prime}-O^{\prime}, S^{\prime}-V-O^{\prime}$, and $S^{\prime}-V^{\prime}-O$ types were devised. A prime (') on a letter indicates that the word the letter symbolizes required additional processing. In the sentence $S^{\prime} \cdot V^{\prime}-0$, for instance, the subject and verb required additional processing, whereas the object did not. To enhance the difference in comprehension time between these sentences and those of the S-V-O type, and thus to facilitate the testing of the proposed models, as explained below, two words of each sentence were selected for additional processing.

Suppose that the processing of $\mathrm{S}, \mathrm{V}$, and $\mathrm{O}$ requires a total time $p$, that integration of the three words requires time $\mathrm{q}$, and that additional processing for an $\mathrm{S}^{\prime}, \mathrm{V}^{\prime}$, or $\mathbf{O}^{\prime}$ requires $3 \mathrm{r}$ each. Then, if the immediate-processing model holds, the total comprehension time for the sentences $S \cdot V^{\prime}-O^{\prime}, S^{\prime}-V-O^{\prime}$, and $S^{\prime} \cdot V^{\prime}-O$ should be approximately the same and should be equal to:

$$
p+q+6 r
$$

However, the continuous-processing model predicts different additional processing times for these sentences, depending on where the $S^{\prime}, V^{\prime}$, or $O^{\prime}$ is embedded in the sentence. Suppose that, at the time of encoding $S^{\prime}$, a reader spends only $r$ units of time over the time spent processing $S$ and postpones the remaining processing time of 2r until V (or $\mathrm{V}^{\prime}$ ) and $\mathrm{O}$ (or $\mathrm{O}^{\prime}$ ) are encountered. Suppose, further, that processing $S^{\prime}$ for the second and third time each requires time $\mathrm{r}$ which is allotted to the time of processing $\mathrm{V}$ (or $\mathrm{V}^{\prime}$ ) and $\mathrm{O}$ (or $\mathrm{O}^{\prime}$ ). If .5r overlaps with the time of processing $V$ (or $V^{\prime}$ ) and the entire $r$ 
overlaps with the time of processing $O$ (or $\mathrm{O}^{\prime}$ ). The additional processing time for $\mathrm{S}^{\prime}$, as compared with that for processing $S$, in a sentence is, then, $r+r / 2$ instead of $3 r$. Similarly, in processing $V^{\prime}$, as compared with processing $\mathrm{V}$, in a sentence, the additional processing of $\mathrm{r}+2 \mathrm{r} / 2$ is required because the remaining processing of $2 \mathrm{r}$ has to be completed at the time of encoding $\mathrm{O}$ (or $\mathrm{O}^{\prime}$ ). If half this time overlaps with the time of encoding $O$ (or $\mathrm{O}^{\prime}$ ), the additional processing time is $\mathrm{r}+1 / 2(2 r)=2 \mathrm{r}$. Finally, in processing $\mathrm{O}^{\prime}$, as compared with processing $\mathrm{O}$, in a sentence, the total additional processing time of $3 \mathrm{r}$ has to be included. In summary, according to the continuous-processing model, the following comprehension times should be obtained:

$$
\begin{aligned}
& p+q+3.5 r \text { for } S^{\prime}-V^{\prime}-O \\
& p+q+4.5 r \text { for } S^{\prime}-V-O^{\prime},
\end{aligned}
$$

and

$$
\mathrm{p}+\mathrm{q}+5 \mathrm{r} \text { for } \mathrm{S}-\mathrm{V}^{\prime}-\mathrm{O}^{\prime}
$$

\section{METHOD}

A variation of the sentence-picture verification task developed by Liu and his associates (Liu, 1980, 1982; Liu \& Chang, 1981; Yu \& Liu, 1982), was used. A trial in the original task consisted of the following sequence of events. (1) Presentation of a first sentence (Sentence 1, whose comprehension and verification were to be assessed independently), was terminated by the subject's keypress after his or her comprehension, thus giving a measure of sentence comprehension. (2) A second sentence (Sentence 2) appeared immediately for a fixed interval (e.g., $800 \mathrm{msec}$ ). (3) Then, a picture (Picture 1) was presented and the subject verified Sentence 1 against Picture 1. (4) Finally, a second picture (Picture 2) appeared and the subject verified Sentence 2 against Picture 2. The purpose of presenting Sentence 2 was to stop the processing of Sentence 1 after the subject's keypress (in order that the comprehension did not continue beyond what was measured) and also to ensure the subject's full comprehension to form a unitary representation (overall meaning) that would resist forgetting when Sentence 2 was processed. The purpose of presenting Picture 2 was to ensure the subject's processing of Sentence 2.

A variation of the original task in the present study consisted of replacing Picture 1 by a sentence, so that, for Event 3 of the sequence, the subject verified Sentence 1 against another sentence designated as Verification-sentence 1 . The obtained verification time of Sentence 1 was then considered to be composed of two parts: the comprehension time of Verification-sentence 1 and the time of verifying Sentence 1 against Verification-sentence 1 that had just been comprehended. If the latter part was assumed to be fairly constant, the obtained verification time of Sentence 1 could be used to infer the magnitude of comprehension time of Verification-sentence 1. The additional processing required for the subject, verb, or object of a sentence could be imposed on the reader by making the subject, verb, or object of Verification-sentence 1 different in word from, but the same in meaning as, the subject, verb, or object of Sentence 1.

\section{Subjects and Materials}

The subjects were 30 undergraduates enrolled in a general psychology course at National Taiwan University. They participated in the experiment to fulfill a course requirement.

All sentences were in Chinese. When translated word-forword into English, they were schematic English sentences. Sentence 1 consisted of "bus" or "swallow" as the subject, "knock against" or "not knock against" as the verb, and "villa" or "kola" as the object. Therefore, there were $2 \times 2 \times 2=8$ kinds of Sentence 1 in all.

The construction of Verification-sentence 1 for a particular instance of Sentence 1 ("Bus knock against villa") is illustrated in Table 1. There were five types of Verification-sentence 1. For the S-V-O type, the same sentence was used as the true sentence, and the false sentences were obtained by replacing the subject, verb, or object with the alternative form because there were two alternatives for each. For the $S^{\prime}-V^{\prime}-O$ type, the true sentence was constructed by replacing the subject and verb with the superordinates ("vehicle" and "hit" in the present example), and the false sentences were constructed by replacing the subject, verb, or object of the true sentence with the alternative superordinate (for the subject or verb) or with the alternative form (for the object). For the $\mathrm{S}^{\prime}-\mathrm{V}-\mathrm{O}^{\prime}, \mathrm{S}-\mathrm{V}^{\prime}-\mathrm{O}^{\prime}$, and $\mathrm{S}^{\prime}-\mathrm{V}^{\prime}-\mathrm{O}^{\prime}$ types, the same rule was used to construct true and

Table 1

Construction of Verification Sentence 1 (Sentence 1 = Bus knock against villa)

\begin{tabular}{cll}
\hline Type & \multicolumn{1}{c}{ True } & \multicolumn{1}{c}{ False } \\
\hline S-V-O & Bus knock against villa & $\begin{array}{l}\text { Swallow knock against villa } \\
\text { Bus not knock against villa } \\
\text { Bus knock against kola } \\
\text { Bird hit villa }\end{array}$ \\
$S^{\prime}-V^{\prime}-0$ & Vehicle hit villa & Vehicle not hit villa \\
& & Vehicle hit kola \\
$S^{\prime}-V^{\prime}-O^{\prime}$ & Vehicle knock against house & Bird knock against house \\
& & Vehicle not knock against house \\
& Vehicle knock against tree \\
$S^{\prime}-V^{\prime}-O^{\prime}$ & Bus hit house & Swallow hit house \\
& & Bus not hit house \\
& & Bus hit tree \\
$S^{\prime}-V^{\prime}-O^{\prime}$ & Bird hit house \\
& Vehicle not hit house \\
& & Vehicle hit tree
\end{tabular}

Note-Each is a translated schematic English sentence. In the original Chinese sentences, the numbers of characters for the subject, verb (affirmative), and object were the same. 
false sentences. Similarly, for each of the other seven sentences used as Sentence 1, the true and false forms of each type of Verification-sentence 1 were constructed.

The set of sentences used as Sentence 2 was "Triangle (rectangle) lack (not lack) a part." The set of pictures used as Picture 2 was a complete (or incomplete) triangle (or rectangle).

\section{Design and Procedure.}

The design was a $5 \times 2 \times 2$ factorial. The first factor refers to type of Verification-sentence 1 (S-V-O, $S^{\prime}-V^{\prime}-O, S^{\prime}-V_{-}-O^{\prime}$, $S-V^{\prime}-O^{\prime}$, or $\left.S^{\prime}-V^{\prime}-O^{\prime}\right)$, the second factor to kind of sentence 1 (affirmative or negative), and the third factor to type of response (true or false). The verification time of Sentence 2 is not analyzed here (see Liu, 1980).

For each Sentence 1 and each type of Verification-sentence 1, there were one true verification sentence and three false verification sentences (see Table 1). To balance the overall true-false ratio, each true verification sentence was presented three times. Therefore, there were altogether $8 \times 5 \times(3+3)=240$ trials. As was explicated at the beginning, a trial consisted of (1) presentation of Sentence 1 for measuring its comprehension time, (2) presentation of Sentence 2 for a fixed interval of $800 \mathrm{msec}$ immediately after the termination of Sentence 1 , (3) presentation of Verification-sentence 1 for measuring the verification time of Sentence 1, and (4) presentation of Picture 2 immediately after the subject verified Sentence 1 for measuring the verification time of Sentence 2.

The presentation and sequencing of the stimulus materials were controlled by an Apple II microcomputer. A C-Plus hardware card with 23,000 Chinese characters implemented was inserted in $I / O$ slot 3 to ensure easy access to Chinese characters.

On each trial, about 2 sec after a "ready" signal spoken by the experimenter, Sentence 1 was presented. To indicate his or her comprehension, the subject pressed the leftmost of five keys arranged horizontally. The next two keys (true and false) were for the verification of Sentence 1, and the rightmost two were for the verification of Sentence 2 . In verifying sentences, the subject was told to press keys quickly without making errors. A random sample of 12 trials was used as a practice block; on each trial, the subject was given feedback. For the 240 experimental trials, administered in random order, no feedback was given.

\section{RESULTS AND DISCUSSION}

With respect to Sentence 1 , the mean comprehension times for affirmatives and negatives were 1.13 and 1.22 sec, respectively. The difference was significant $[t(29)=$ $5.64, \mathrm{p}<.01]$.

The main interest of the present experiment was in the mean verification times of Sentence 1 when the five types of Verification-sentence 1 were used. The obtained results are presented in Table 2 . As is apparent from Table 2, the pattern of verification times for true affirmatives (TA), false affirmatives (FA), true negatives (TN), and false negatives (FN) obtained for the S-V-O type is quite different from the patterns obtained for the other types of Verification-sentence 1. Starting from the second row in Table 2, reading down the TA, FA, and FN columns, one finds a steady increase in verification time. With respect to the TN column, except for the case of $\mathbf{S}^{\prime} \cdot \mathbf{V}^{\prime}-\mathrm{O}^{\prime}$, the verification times were almost constant for the remaining types of Verification-sentence 1. An analysis of variance showed that the type of Verification-sentence 1 was a significant source of variance $[\mathrm{F}(4,116)=14.42, \mathrm{MSe}=.0362, \mathrm{p}<.01]$. Affirmatives were verified faster than negatives $[F(1,29)=19.00$, MSe $=.0199, \mathrm{p}<.01]$. True responses did not differ from false responses $(F<1)$. However, kind of Sentence 1 (affirmative vs. negative) interacted with type of response $[\mathrm{F}(1,29)=5.93, \mathrm{MSe}=.0460, \mathrm{p}<.05]$. Another significant interaction was obtained between type of Verification-sentence 1 and type of response $[\mathrm{F}(4,116)=5.17, \mathrm{MSe}=.0340, \mathrm{p}<.01]$.

As for the obtained errors, an analysis of variance showed that more errors were found in false responses than in true responses $[\mathrm{F}(1,29)=14.36, \mathrm{MSe}=.314$, $\mathrm{p}<.01]$. Type of Verification-sentence 1 was also a significant source of variance $[\mathrm{F}(4,116)=4.83, \mathrm{MSe}=.006$, $p<.01$ ] . It can be seen from Table 2 that this effect is mainly due to more errors made for the $S^{\prime} \cdot V^{\prime} \cdot O^{\prime}$ type than for the other types. All other effects were not significant.

Although a significant interaction was found between type of Verification-sentence 1 and type of response, according to Table 2 this interaction was due mainly to an exceptional pattern of verification times for the S-V-O type. Therefore, as far as the remaining types of Verification-sentence 1 are concerned, the variable of verification (kind of Sentence 1 and type of response) can be considered to be additive with respect to type of Verification-sentence 1 . If so, the verification time obtained for each of these types of Verification-sentence 1 should consist of two parts: the comprehension time of Verification-sentence 1 and a constant pure verification time of Sentence 1. In other words, the mean verification times of Table 2, except those for the S-V-O type, can be considered to reflect relative comprehension times of the various types of Verification-sentence 1. Except for the entries in the TN column, the mean verification times of Table 2 show the inequalities

$$
S^{\prime} \cdot V^{\prime}-O<S^{\prime}-V \cdot-O^{\prime}<S-V^{\prime}-O^{\prime}
$$

Table 2

Mean Verification Time (in Seconds) and Percentage of Errors (PE)

\begin{tabular}{ccccccccc}
\hline Type & TA & PE & FA & PE & TN & PE & FN & PE \\
\hline S-V-O & 1.11 & 5.50 & 1.25 & 21.10 & 1.23 & 3.30 & 1.31 & 21.90 \\
S'-V'-O & 1.11 & 2.20 & 1.11 & 20.80 & 1.23 & 3.80 & 1.08 & 21.10 \\
S'-V-O' & 1.17 & 6.30 & 1.17 & 20.20 & 1.22 & 3.60 & 1.16 & 21.30 \\
S-V'-O' & 1.17 & 7.50 & 1.23 & 19.70 & 1.22 & 5.80 & 1.25 & 22.70 \\
S'-V'-O' $^{\prime}$ & 1.28 & 5.80 & 1.28 & 25.50 & 1.40 & 5.20 & 1.28 & 28.00 \\
\hline
\end{tabular}


which are consistent with the predicted relative magnitudes of Equations 2, 3, and 4. The present finding thus favors the continuous-processing model over the commonly held immediate-processing model.

The reason that the verification pattern for the S-V-O type was different from the patterns for the other types may be explained by the different strategies used. With respect to the S-V-O type, the words used for Sentence 1 and Verification-sentence 1 in the "true" case were identical. Therefore, the subject proceeded to construct a unitary representation also for a negative sentence in comprehending Verification-sentence 1, which resulted in the TN < FN ordering (Liu, 1980). When some words were replaced by the superordinates in Verification-sentence 1 of the types other than the S-V-O type, the subject tended to look for places of mismatches. This explains why the $\mathrm{FN}<\mathrm{TN}$ ordering was obtained in many cases: When a mismatch happened in the first or second words, it would be unnecessary to process the entire sentence in order to arrive at a deci- sion. This also explains why high percentages of errors were obtained for false responses.

\section{REFERENCES}

Just, M. A., \& Carpenter, P. A. (1980). A theory of reading: From eye fixation to comprehension. Psychological Review, 87, 329-354.

LIU, I. (1980). Separating comprehension from the verification process. Memory \& Cognition, 8, 75-83.

LiU, I. (1982). The retroactive effect of argument overlap on sentence retrieval. American Journal of Psychology, 95, 85-101.

Liv, I., \& Chang, S. (1981). Short-term memory of affirmative and negative sentences. Acta Psychologica Taiwanica, 23, 131-136.

Navon, D., \& Gopher, D. (1979). On the economy of the humanprocessing system. Psychological Review, 86, 214-255.

YU, P., \& LIU, I. (1982). Applicability of the model of Chinese sentence comprehension to English sentences. Acta Psychologica Taiwanica, 24, 59-63.

(Manuscript received for publication October 11, 1983.) 